\title{
Proximal Predictors of Long-Term Discontinuance with Noninsulin Antihyperglycemic Agents
}

\author{
Bruce C. Stuart, PhD; Xian Shen, MS; Charlene C. Quinn, RN, PhD, FAAN; \\ Nicole Brandt, PharmD; Pamela Roberto, MPP; F. Ellen Loh, BSPharm, MBA; \\ Franklin Hendrick, BS; Caroline Kim, BS; Xingyue Huang, PhD; and Swapnil Rajpathak, MD
}

\begin{abstract}
BACKGROUND: Noninsulin antihyperglycemic agents (NAAs) are the mainstay of treatment for type 2 diabetes, yet persistence in NAA use is suboptimal in many diabetes patients. Most of the research on NAA discontinuance has focused on sociodemographic characteristics and general health status, but such factors are inherently limited in explaining dynamic events such as discontinuance.
\end{abstract}

OBJECTIVE: To assess the relative importance of static and proximal dynamic factors in explaining long-term NAA discontinuance among Medicare beneficiaries with diabetes.

METHODS: Two sets of probability models were estimated to predict NAA discontinuance as a function of static variables (age, sex, race, original reason for Medicare entitlement, low-income subsidy and dual Medicare/ Medicaid eligibility status, and disease burden) and 21 dynamic factors capturing month-by-month changes in drug use, health status, and use of medical services leading up to discontinuance (defined as month 0 ) and the previous 4 months (designated months -1 to -4 ) among 71,619 patients with diabetes enrolled in Medicare Part D plans in 2006-2008.

RESULTS: Static variables explained just $1.2 \%$ of the variance in probability of NAA discontinuance compared with $14 \%$ for all variables combined. Key time-related predictors of NAA discontinuance included discontinuation with angiotensin-converting enzyme inhibitors/angiotensin receptor blockers (ACEls/ARBs) and statins, hypoglycemia, NAA usage gaps, insulin use, and discharge from hospitals and skilled nursing facilities (SNFs). The strongest significant predictors $(P<0.05)$ of NAA discontinuance were discontinuation with statins and ACEIs/ARBs in month 0 (predicted probabilities of $37 \%$ and $34 \%$, respectively). Other variables that significantly increased the probability of NAA discontinuance by $10 \%$ or more were hypoglycemia in month $0(14 \%)$ and month $-1(17 \%)$, discontinuance with ACEls/ARBs in months $-1(15 \%)$ and $-2(10 \%)$, discontinuance with statins in month $-1(13 \%)$, and insulin use in month $0(12 \%)$. Experiencing a previous gap in NAA therapy was associated with higher likelihood of discontinuance if the gap occurred in month $-2(10 \%)$ or month $-4(6 \%)$, but a gap in therapy in month -1 actually reduced the likelihood of discontinuance by $13 \%$. Discharge from a hospital or SNF was consistently associated with higher probabilities of NAA discontinuance ranging between $4 \%$ and $10 \%$, with higher probabilities occurring closer to month 0 .

CONCLUSIONS: A cascade of dynamic changes preceding discontinuance with NAA therapy among Medicare Part $D$ enrollees with diabetes was observed between 2006 and 2008. Understanding that lack of persistence in drug use is a dynamic rather than a static phenomenon opens up new avenues for investigating and ultimately improving adherence behavior in the elderly.

\section{J Manag Care Spec Pharm. 2016;22(9):1019-27}

Copyright $\odot 2016$, Academy of Managed Care Pharmacy. All rights reserved.

\section{What is already known about this subject}

Discontinuation with noninsulin antihyperglycemic agents (NAA) is a common phenomenon among patients with type 2 diabetes mellitus.

Known risk factors for NAA discontinuance include younger age, nonwhite race, low income, high comorbidity burden, and other non-time varying characteristics that cannot, in and of themselves, predict the timing of a dynamic event such as drug discontinuance.

Certain dynamic factors associated with NAA discontinuance (hypoglycemia, hospitalization, and emergency department visits) have been studied but not in a time-to-event analytic framework.

\section{What this study adds}

A month-to-discontinuation model was developed that identified timed factors hypothesized to predict long-term NAA discontinuation derived from Medicare enrollment and claims data.

Dynamic factors proximal to discontinuation were shown to be far better predictors than static factors typically studied in the adherence literature.

Analyzing medical as well as prescription drug claims data can help health plans and clinicians identify circumstances when patients are at elevated risk of drug discontinuation.

$\mathrm{N}$ oninsulin antihyperglycemic agents (NAAs) are the mainstay of treatment for type 2 diabetes mellitus (T2DM). Appropriate NAA treatment substantially reduces the risk of microvascular disease and other diabetes complications. ${ }^{1-3}$ NAAs are prescribed to T2DM patients who are unable to control their glycemic levels through diet or exercise. Treatment typically begins with metformin or a sulfonylurea and may be augmented with a second NAA (alpha-glucosidase inhibitor, amylinomimetic, thiazolidinedione, meglitinide, dipeptidyl peptidase-4, or incretin mimetic) or insulin if the initial agent provides inadequate control. Treatment generally lasts a lifetime. Yet, persistence with NAAs is suboptimal in many diabetes patients with rates of discontinuance reaching $50 \%$ or more within 1.5 years of initiation. ${ }^{4,5}$ Reasons for poor persistence are not well understood. Most research in this area has focused on demographic characteristics 
and general health status, ${ }^{5-13}$ but static factors alone cannot explain dynamic events such as discontinuance. A few studies have examined specific proximal factors associated with NAA discontinuance, such as medication experience, ${ }^{4}$ type of NAA prescribed, ${ }^{14}$ presence of hypoglycemic events, ${ }^{14-15}$ refill gaps, ${ }^{5,6}$ hemoglobin Alc measures, ${ }^{16}$ distribution channel, ${ }^{17}$ and medication cost. ${ }^{5,6,18}$ But to date, no comprehensive study has assessed how dynamic factors combine to explain NAA discontinuance, particularly for long-term discontinuers. As a result, clinicians are ill equipped to diagnose triggers for discontinuance and thus miss potentially important intervention opportunities. The objective of this study was to assess the relative importance of static and proximal dynamic factors in explaining long-term NAA discontinuance among diabetes patients enrolled in Medicare Part D plans in 2006-2008.

\section{Methods}

\section{Data Source}

Data were obtained from the Chronic Condition Data Warehouse (CCW) prepared by Buccaneer, a data contractor for the Centers for Medicare \& Medicaid Services (CMS). The dataset comprised a random 5\% sample of the Medicare population in 2006, which was then tracked through 2008. The data files included basic demographic and program enrollment information plus claims for inpatient and outpatient services as well as prescription drugs. The Part A and B claims include International Classification of Diseases, Ninth Revision, Clinical Modification (ICD-9-CM) diagnostic codes used to identify diabetes and other diseases. The Part D claims files include National Drug Code (NDC) numbers that were used to identify drugs in classes of interest. The CCW files include a variable for first recorded diabetes diagnosis dating back to 1999.

\section{Study Sample}

The sample was selected based on the following inclusion criteria: (a) continuously enrolled in Medicare Part A, Part B, and Part D fee-for-service prescription drug plans from January 1, 2006, through December 31, 2008, or death; (b) diagnosed with T2DM before 2006 (ICD-9-CM codes 250.xx, 357.2, 362.01, 362.02 , and 366.41 on any hospital claim or 2 outpatient or carrier claims); and (c) filled at least 2 NAA prescriptions during the study period.

From this base population, 2 cohorts were identified: longterm NAA discontinuers and continuing NAA users. Long-term discontinuers were identified by using a 2-step process. First, prescription records from the base population were reviewed to identify all NAA users with a minimum of 2 fills for one or more different NAAs in 2007 followed by an absence of any NAA use over the next 12 months. The last month with NAA days supply was defined as the index month (month 0 ). The 11 months previous to the index month were designated as the baseline period during which potential proximal factors associated with discontinuance were assessed. Second, beneficiaries who died within 5 months of their index months were excluded. The rationale for this exclusion is that impending death was likely the proximal cause for discontinuance, which would typically be considered clinically appropriate for therapies with extended time to benefit such as NAAs. Individuals were retained who died 6 months or more after their index dates on the presumption that the proximal reasons for NAA discontinuance were factors other than impending death. The cutpoint of 6 months is admittedly arbitrary and is based on the predicted survival threshold used in the Medicare hospice benefit. In 2008, 40\% of Medicare beneficiaries who died used hospice services, ${ }^{19}$ so it is reasonable to assume that physicians were familiar with the drug reduction regimens used in the program.

Next, a comparison cohort of continuing NAA users was identified. Continuing users were required to have a minimum of 2 NAA fills in their baseline and follow-up periods. We randomly assigned continuing users an index month through an algorithm that equated the distribution of index months for the 2 cohorts (this was designed to reduce any bias associated with seasonal factors that might influence drug discontinuation) and eliminated anyone who died in months 0 to 5. NAA users who fit neither the definition of a long-term discontinuer nor a continuing user were excluded.

\section{Measures}

We specified 28 variables from Medicare enrollment records and claims files that were hypothesized to influence persistence or discontinuance with NAA therapy. First were 7 static variables: age, gender, race, original reason for Medicare entitlement, low-income subsidy (LIS) status, dual eligibility, and annual counts of chronic conditions in the CMS risk adjuster known as RxHCC (prescription drug hierarchical chronic conditions), all measured in the baseline year of 2006. The original reason for Medicare entitlement included age 65, Social Security Disability Insurance (SSDI), and end-stage renal disease (ESRD). The SSDI and ESRD indicators captured beneficiaries with high disease burden and generally lower socioeconomic status. Dual Medicare/Medicaid eligibility and receipt of LIS captured the poorest Medicare beneficiaries. The RxHCC measure served as a Medicare-specific comorbidity index. We hypothesized that being older and nonwhite with lower socioeconomic status and higher disease burden would be associated with a greater probability of NAA discontinuance.

We specified 4 categories of potential dynamic predictors of NAA discontinuance: (1) drug use, (2) diabetes tests and preventive measures, (3) services indicating treatment for diabetes complications and comorbidities, and (4) Medicare use and spending. All variables were measured during the index month and for each of the preceding 4 months (in preliminary analyses, we evaluated events extending back 11 months previous to the index month, but because observations before month -4 were never consistently predictive of discontinuation, they are not reported here). 


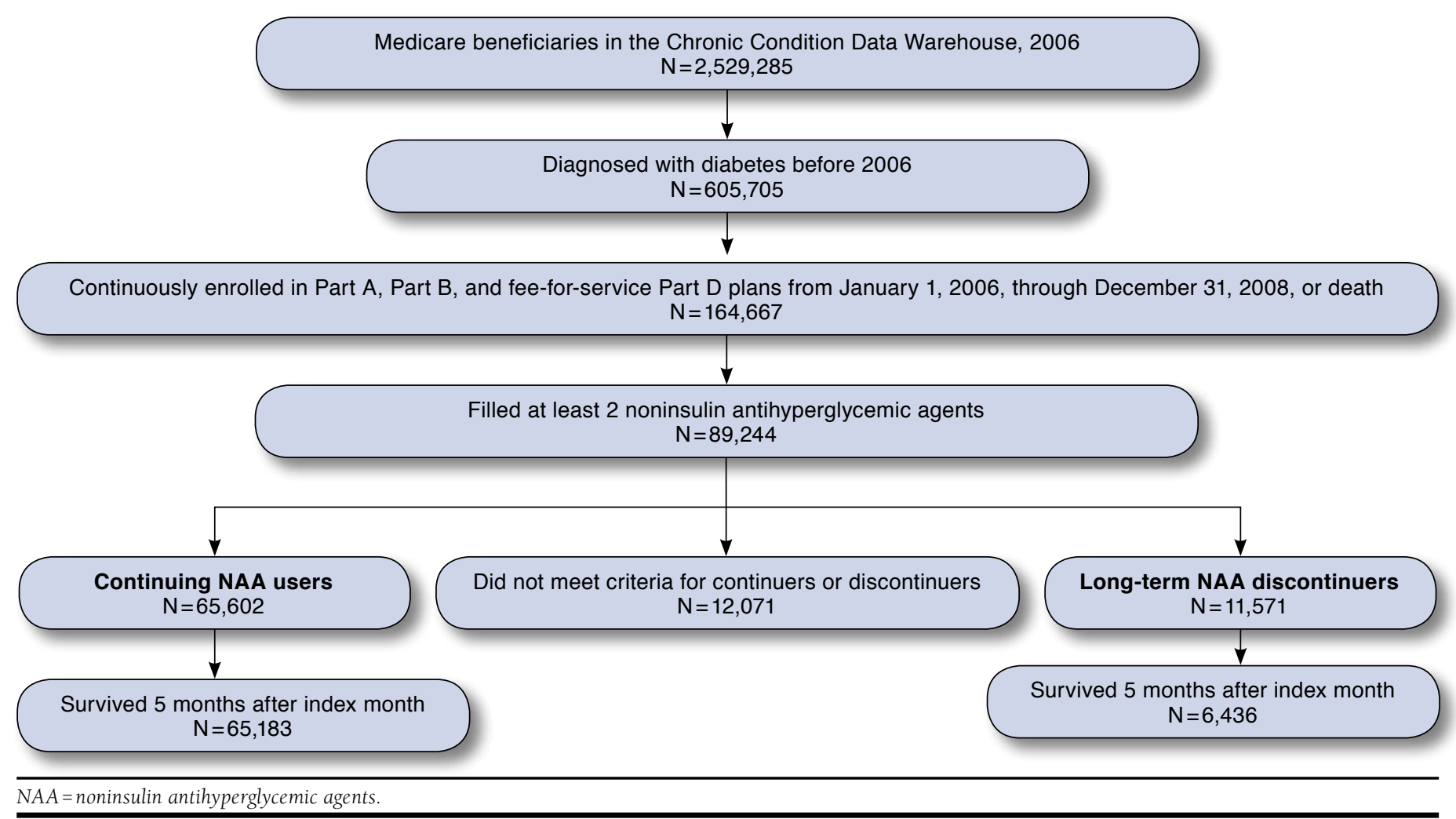

The NAA drugs included in the analysis were prescription fills for metformin, sulfonylureas, alpha-glucosidase inhibitors, thiazolidinediones, dipeptidyl peptidase-4 inhibitors, meglitinides, and incretin mimetics. Antidiabetic drug predictor variables included first month of observed NAA use, NAA usage gaps defined as 30 days or more with no NAAs on hand bracketed by days with NAAs, monthly counts of different NAAs filled, any insulin fill, discontinuation with concomitant therapy for hypertension including angiotensin-converting enzyme inhibitors (ACEIs) and angiotensin receptor blockers (ARBs), and discontinuance with concomitant therapy for treating hyperlipidemia (statins). We hypothesized that NAA usage gaps, insulin fills, and discontinuation with ACEIs/ARBs and statins would be positively associated with NAA discontinuation, while early NAA initiation and multiple NAAs would reduce the probability of NAA discontinuation.

Monthly indicators were created for the following diabetes tests and preventive measures: tests for Alc, fasting blood glucose, and diabetic eye exam. These variables were included for exploratory purposes without signed hypotheses (e.g., more frequent tests could indicate either worsening condition or good diabetes management).
The next set of variables was intended to capture differences in diabetes severity between the 2 study cohorts: uncontrolled diabetes (ICD-9-CM code 250.x2); short-term diabetes complications (ICD-9-CM codes 250.1x, 250.2x, and 250.3x); longterm diabetes complications (ICD-9-CM codes $250.4 \mathrm{x}, 250.5 \mathrm{x}$, 250.6x, 250.7x, 250.8x, and 250.9x); hypoglycemia (ICD9-CM code 251.2); ESRD (ICD-9-CM code 585.6); and cancer (ICD-9-CM codes 14.xx to 209.xx, excluding skin cancer). We hypothesized that hypoglycemia would increase the probability of NAA discontinuance. The other severity indicators were included as exploratory factors.

The final set of monthly variables included indicators for hospital discharge, skilled nursing facility (SNF) discharge, hospice admission, number of emergency department visits, number of physician visits, number of unique physicians seen in the month, and monthly values for Part A, B, and D spending. We hypothesized that all variables except Part D spending would be associated with increased likelihood of NAA discontinuance.

\section{Statistical Analysis}

Factors associated with long-term NAA discontinuance were estimated, using ordinary least squares (OLS) linear probability models and logistic regression models. Although OLS is not 


\begin{tabular}{|c|c|c|c|}
\hline Characteristics & $\begin{array}{l}\text { NAA } \\
\text { Discontinuers } \\
(n=6,436)\end{array}$ & $\begin{array}{c}\text { NAA } \\
\text { Continuing } \\
\text { Users } \\
(\mathrm{n}=65,183)\end{array}$ & $P$ Value \\
\hline Age in years [SD] & $71.3[13.2]$ & $70.5[12.1]$ & $<0.001$ \\
\hline Sex, \% & & & 0.388 \\
\hline Male & 33.6 & 34.2 & \\
\hline Female & 66.4 & 65.8 & \\
\hline \multicolumn{3}{|l|}{ Race, \% } & $<0.001$ \\
\hline White & 69.8 & 73.7 & \\
\hline Black & 19.2 & 14.9 & \\
\hline Other and unknown & 11.0 & 11.4 & \\
\hline \multicolumn{3}{|c|}{ Original reason for Medicare entitlement, \% } & $<0.001$ \\
\hline Old age & 59.5 & 64.6 & \\
\hline SSDI/ESRD & 40.5 & 35.4 & \\
\hline \multicolumn{3}{|l|}{ Low-income subsidy, \% } & $<0.001$ \\
\hline Yes & 72.0 & 66.2 & \\
\hline No & 28.1 & 33.8 & \\
\hline \multicolumn{3}{|l|}{ Dual eligibility status, $\%$} & $<0.001$ \\
\hline Yes & 68.2 & 62.6 & \\
\hline No & 31.8 & 37.4 & \\
\hline RxHCC counts [SD] & $8.6[3.8]$ & $7.3[3.3]$ & $<0.001$ \\
\hline \multicolumn{4}{|c|}{$\begin{array}{l}\text { ESRD = end-stage renal disease; } N A A=\text { noninsulin antihyperglycemic agents; } \\
\text { RxHCC = Prescription Drug Hierarchical Condition Category; } S D=\text { standard } \\
\text { deviation; SSDI = Social Security Disability Insurance. }\end{array}$} \\
\hline
\end{tabular}

traditionally used to estimate models with binary outcomes, it has several advantages in the context of this analysis. First, because we had no a priori knowledge of the true functional form of the relationships being investigated, we selected linear relationships as the simplest alternative. Second, we wished to provide a measure of how each variable contributed to explained variance and that is directly possible only with OLS (through partial and cumulative $\mathrm{R}^{2}$ measures). Third, the results from such tests are far more intuitive-the OLS model coefficients are read as marginal probabilities-than those derived from standard logistic models. A final advantage of OLS over nonlinear models is that parameter estimates are additive, thereby making it easy to identify combinations of effects that are most predictive of drug discontinuation. Two drawbacks to OLS models are that the predicted probabilities are not constrained to the unit interval, and the OLS errors are heteroskedastic. However, as Wooldridge (2013) notes in his classic econometrics text,20 finding small numbers of predicted probabilities outside the unit interval is seldom problematic, and calculating robust standard errors controls for heteroscedasticity.

The initial OLS model estimated the probability that a study subject was classified as an NAA discontinuer as a function of the 7 static variables previously described. Next, a forward selection model was used that included static variables plus 5 monthly values for each dynamic factor. The variable with the lowest $P$ value was entered first, followed sequentially by the variable with next lowest $P$ value, and so on, until no additional variable met the minimum criterion of $P<0.05$. Results from otherwise identical models estimated using logistic regression are provided in Appendices A and B (available in online article).

\section{Results}

\section{Sample Characteristics}

Based on the study selection criteria (Figure 1), we identified 6,436 long-term NAA discontinuers and 65,183 continuing NAA users. It is worth noting that the exclusion criteria for impending death (died in months 0-5) had a much greater impact on delineating discontinuers compared with continuing users. As expected, NAA use is rare immediately preceding death. Baseline characteristics of the 2 cohorts are presented in Table 1 . The distribution of age and gender was very similar, but discontinuers were less likely to be white (70\% vs. $64.6 \%$ ) and were more likely to have entered Medicare through SSDI or ESRD (40.5\% vs. 35.4\%). The discontinuer cohort also had higher proportions of LIS recipients and dual eligibles and carried a significantly heavier disease burden measured by RxHCC counts (8.6 vs. 7.3),

Dynamic characteristics of the 2 cohorts in the index month (0) and previous 4 months (-1 through -4$)$ are summarized in Table 2. The first set of measures highlights differences in drug use. As hypothesized, NAA discontinuers exhibited much more volatile behavioral patterns compared with continuing users. In particular, discontinuers were more than twice as likely to experience gaps in NAA use before their index dates. They were less likely to use multiple NAAs but were much more likely to use insulin (e.g., 30.7\% of discontinuers took insulin in month -4 rising to $39.2 \%$ in the index month, whereas insulin use among continuing users averaged just $15 \%$ with little change over time). Discontinuation with other ACEIs/ARBs and statins was relatively rare in both groups but spiked to nearly $5 \%$ among NAA discontinuers in the index month.

NAA discontinuers experienced strikingly higher rates of diabetes complications and comorbidities compared with continuing users. This was particularly apparent for ESRD, short-term diabetes complications, and hypoglycemia, albeit the prevalence of such complications was low in both groups. Similar patterns were evident for the Medicare use and spending variables shown in the bottom panel of Table 2. On a monthly basis, discontinuers had twice the number of emergency department visits and different physicians visited, 3 times the number of hospital discharges, and 4 times the number of SNF discharges. More contacts with the health system resulted in much higher spending on Medicare services by NAA discontinuers, with a marked spike in spending in the month immediately before discontinuation. 
Proximal Predictors of Long-Term Discontinuance with Noninsulin Antihyperglycemic Agents

TABLE 2 Differences in Dynamic Characteristics by NAA Continuance Status and Month Previous to Index Month

\begin{tabular}{|c|c|c|c|c|c|c|c|c|c|c|}
\hline \multirow[b]{3}{*}{ Dynamic Variables } & \multirow{2}{*}{\multicolumn{4}{|c|}{$\begin{array}{l}\text { NAA Discontinuers }(n=6,436) \\
\text { Month Previous to Index Month }\end{array}$}} & \multirow{3}{*}{$\begin{array}{c}\text { Index } \\
\text { Month }\end{array}$} & \multirow{2}{*}{\multicolumn{4}{|c|}{$\begin{array}{c}\text { NAA Continuing Users }(n=65,183) \\
\text { Month Previous to Index Month }\end{array}$}} & \multirow{3}{*}{$\begin{array}{c}\begin{array}{c}\text { Index } \\
\text { Month }\end{array} \\
0\end{array}$} \\
\hline & & & & & & & & & & \\
\hline & -4 & -3 & -2 & -1 & & -4 & -3 & -2 & -1 & \\
\hline \multicolumn{11}{|l|}{ Drug use variables } \\
\hline NAA usage gap, $\%$ & 15.89 & 15.78 & 17.22 & 4.36 & - & 6.39 & 6.39 & 5.97 & 3.95 & - \\
\hline Different NAAs filled, $\mathrm{n}$ & 0.7 & 0.8 & 0.6 & 0.8 & - & 1.0 & 1.0 & 1.0 & 1.1 & - \\
\hline Any insulin use, \% & 30.73 & 31.40 & 32.78 & 35.90 & 39.21 & 15.33 & 15.53 & 15.68 & 15.97 & 16.21 \\
\hline Discontinuation of ACEIs/ARBs, \% & 0.61 & 0.65 & 1.04 & 1.54 & 4.93 & 0.27 & 0.31 & 0.33 & 0.33 & 0.43 \\
\hline Discontinuation of statins, $\%$ & 0.36 & 0.62 & 0.73 & 1.09 & 4.81 & 0.28 & 0.26 & 0.29 & 0.31 & 0.35 \\
\hline \multicolumn{11}{|c|}{ Diabetes tests and other preventive measures, $\%$} \\
\hline Alc test & 17.95 & 18.38 & 17.17 & 21.16 & 19.52 & 18.48 & 18.28 & 18.69 & 19.05 & 18.72 \\
\hline Fasting blood glucose test & 3.70 & 3.89 & 4.36 & 4.76 & 4.81 & 3.73 & 3.72 & 3.73 & 3.93 & 3.73 \\
\hline Eye exam & 6.68 & 6.24 & 6.02 & 5.95 & 6.27 & 6.96 & 6.94 & 6.89 & 7.05 & 6.98 \\
\hline \multicolumn{11}{|c|}{ Diabetes complications and comorbidities, ${ }^{\mathrm{a}} \%$} \\
\hline Uncontrolled diabetes & 9.76 & 10.13 & 11.10 & 13.22 & 11.80 & 8.38 & 8.39 & 8.52 & 8.73 & 9.02 \\
\hline Short-term diabetes complications & 0.47 & 0.36 & 0.50 & 0.58 & 0.45 & 0.27 & 0.29 & 0.27 & 0.29 & 0.29 \\
\hline Long-term diabetes complications & 7.08 & 7.50 & 7.46 & 9.57 & 8.50 & 5.43 & 5.32 & 5.57 & 5.47 & 5.77 \\
\hline Hypoglycemia & 0.70 & 0.76 & 0.93 & 1.40 & 1.15 & 0.18 & 0.21 & 0.20 & 0.20 & 0.20 \\
\hline ESRD & 3.27 & 3.44 & 3.55 & 3.70 & 3.89 & 1.04 & 1.05 & 1.07 & 1.10 & 1.11 \\
\hline Cancer & 2.07 & 1.87 & 2.05 & 2.04 & 2.15 & 1.85 & 1.93 & 1.94 & 1.91 & 1.95 \\
\hline \multicolumn{11}{|l|}{ Medicare utilization and spending } \\
\hline Hospital discharge, $\%$ & 9.40 & 8.83 & 10.91 & 13.37 & 10.17 & 3.53 & 3.62 & 3.55 & 3.62 & 3.72 \\
\hline SNF discharge, $\%$ & 2.01 & 2.26 & 2.26 & 3.39 & 2.16 & 0.50 & 0.52 & 0.55 & 0.48 & 0.50 \\
\hline Hospice admission, \% & 0.12 & 0.09 & 0.08 & 0.03 & 0.54 & 0.04 & 0.04 & 0.04 & 0.01 & 0.10 \\
\hline ED visits, $n$ & 0.2 & 0.2 & 0.2 & 0.2 & 0.2 & 0.1 & 0.1 & 0.1 & 0.1 & 0.1 \\
\hline Unique physicians seen, $\mathrm{n}$ & 2.1 & 2.1 & 2.3 & 2.5 & 2.2 & 1.2 & 1.2 & 1.2 & 1.2 & 1.2 \\
\hline Part A \& B spending, \$ & $2,225.51$ & $2,403.83$ & $2,603.14$ & $3,007.58$ & $2,240.41$ & 934.02 & 939.62 & 924.26 & 921.49 & 922.47 \\
\hline Part D spending, \$ & 403.35 & 420.29 & 395.33 & 434.64 & 383.69 & 357.41 & 357.82 & 364.02 & 369.03 & 372.70 \\
\hline
\end{tabular}

\section{Multivariate Findings}

Results from the OLS model predicting NAA discontinuation as a function of static variables only are shown in Table 3. All variables in this model except sex and other race were statistically significant predictors at $P<0.05$, but the effects were small. The biggest difference was a $2.6 \%$ higher probability of discontinuance by blacks compared with whites. Beneficiaries whose Medicare entitlement was because of age were $1.5 \%$ less likely to discontinue NAAs, compared with those who entered the program through SSDI or ESRD. Being an LIS recipient increased risk of discontinuance by $0.9 \%$, about the same amount as each additional RxHCC condition. Total explained variance attributable to all model variables was just $1.2 \%$. Results from an identically specified logistic regression (see Appendix A, available in online article) were similar in direction and magnitude. The $C$ statistic of 0.6 indicated that the combined predicted probability of NAA discontinuance was scarcely better than chance alone.
The multivariate OLS stepwise regression results are presented in Table 4. The variables are listed in the order in which they entered the model. The last column shows the cumulative $\mathrm{R}^{2}$ through step 50 , after which no variable was statistically significant at $P<0.05$. The adjusted probabilities together with robust standard errors (which corrected for heteroscedasticity) are taken from the final 50-step equation. Findings from the logistic stepwise regression are shown in Appendix B (available in online article). These results are very similar to those generated by the OLS model in terms of parameter signs, significance levels, and relative order of magnitude, albeit the order in which variables entered the logistic model differed somewhat from the OLS version.

It is immediately apparent that dynamic characteristics explain far more of the total variance in the likelihood of discontinuance (14\%), compared with static variables (1.2\%). The strongest individual predictors of NAA discontinuance were discontinuation with statins and ACEIs/ARBs in the index month (predicted probabilities of 37\% and 34\%, 


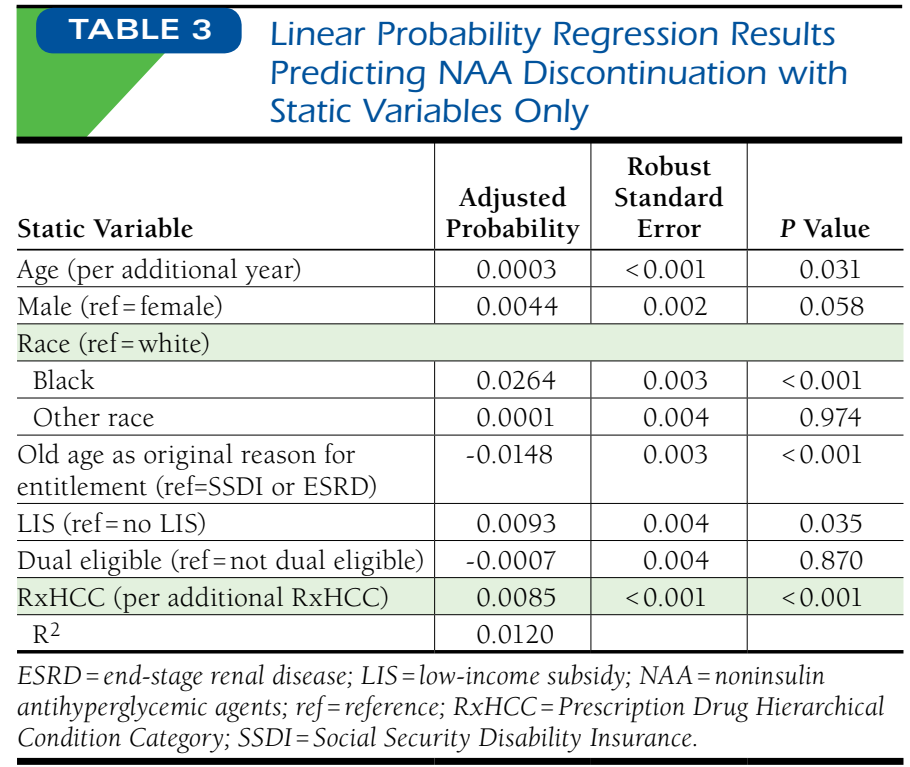

respectively). Other variables that increased the probability of NAA discontinuance by $10 \%$ or more were hypoglycemia in month 0 (14\%) and month -1 (17\%), discontinuance with ACEIs/ARBs in month -1 (15\%) and month -2 (10\%), discontinuance with statins in month -1 (13\%), and insulin use in the index month (12\%). Experiencing a previous gap in NAA therapy was associated with higher likelihood of discontinuance if the gap occurred in month -2 (10\%) or month -4 (4\%), but a gap in therapy in month -1 actually reduced the likelihood of discontinuance by $13 \%$. Discharge from a hospital or SNF was consistently associated with higher probabilities of NAA discontinuance ranging between $4 \%$ and $10 \%$, with higher probabilities occurring in months closer to the index month. The model contained numerous other statistically significant predictors of long-term NAA discontinuance, but the effects were generally small and occasionally inconsistent in signs.

Two points should be kept in mind when interpreting these results. First, the unadjusted probability of long-term NAA discontinuation in this study sample was just $9 \%$, so even the largest estimated marginal effects increased the absolute probability of discontinuation by just a few percentage points. Second, although parameter estimates in OLS equations are technically additive, some variable combinations are ruled out by definition (e.g., it is only possible to discontinue ACEIs/ ARBs or statins once, and gaps in NAA therapy cannot occur in contiguous months). Nonetheless, there are several combinations of predictive factors, such as discontinuation with ACEIs/ ARBs and statins, hypoglycemia, and institutional transitions, that together more than double the likelihood of long-term discontinuation.

\section{Discussion}

Several aspects of the results warrant discussion. First, as hypothesized, a model containing only static predictors explained very little of the total variance in long-term NAA discontinuation in a nationally representative sample of diabetes patients enrolled in Medicare Part D plans $\left(R^{2}=0.012\right)$, compared with a model that included time-dependent proximal predictors $\left(\mathrm{R}^{2}=0.14\right)$. The earliest a static variable entered the stepwise model was step 19 (LIS). "Black" entered the equation at step 33. Although being an LIS recipient or black may (slightly) increase the risk of long-term drug discontinuance, the vast majority of NAA users who shared these characteristics were not discontinuers, and those who were must logically have responded to some time-related stimulus (or lack thereof) that influenced when they stopped taking their medications. This is not to suggest that clinicians and policymakers should forgo focusing on individual characteristics associated with higher risk of drug discontinuance, just that the focus should be combined with information on dynamic circumstances for which the chances of discontinuation are highest.

Second, relatively few dynamic variables accounted for almost all of the variance in long-term NAA discontinuance. The first 5 variables in the stepwise regression accounted for over $70 \%$ of the total explained variance, and 5 of the first 7 variables had coefficient values (adjusted probabilities) exceeding 10 percentage points. Key time-related predictors of NAA discontinuance in the model included discontinuation with ACEIs/ARBs and statins, hypoglycemia, NAA usage gaps, insulin use, and discharge from hospitals and SNFs.

Third, the time to estimated effect was short. We originally estimated the effect of 21 dynamic variables up to 11 months before the index month but found that values more than 5 months before the index month had little or no predictive power. But, even when the analysis was restricted to a shorter time horizon, the strongest effects were observed either in the index month itself or the 2 months immediately preceding it. This observation suggests that prognostic tools designed to identify circumstances under which high-risk patients are most likely to have difficulty remaining on their medications should focus on short-run targets.

\section{Limitations}

This study has a number of important limitations that suggest avenues for future research, with potentially high returns in improving medication adherence among older people with serious chronic disease. Perhaps the most important limitation is that this model is deterministic rather than prognostic. We compared known discontinuers with known continuing users for the expressed purpose of being able to go back in time to identify not only which circumstances were most strongly associated with discontinuation but how that association changed as the discontinuation decision neared. 
TABLE 4 Stepwise Linear Probability Regression Results Predicting NAA Discontinuation as a Function of Static and Dynamic Variables

\begin{tabular}{|c|c|c|c|c|}
\hline Step & Variable & Parameter Estimate & Robust Standard Error & Model $\mathrm{R}^{2}$ \\
\hline 1 & Number of NAAs filled month -2 & -0.02 & $<0.01$ & 0.02 \\
\hline 2 & Insulin use month 0 & 0.12 & 0.01 & 0.05 \\
\hline 3 & Number of unique physicians seen month -1 & 0.00 & $<0.01$ & 0.06 \\
\hline 4 & Discontinuance of statins month 0 & 0.37 & 0.02 & 0.08 \\
\hline 5 & Discontinuance of ACEIs/ARBs month 0 & 0.34 & 0.02 & 0.10 \\
\hline 6 & NAA usage gap month -2 & 0.10 & 0.01 & 0.10 \\
\hline 7 & NAA usage gap month -1 & -0.13 & 0.01 & 0.11 \\
\hline 8 & Number of NAAs filled month -1 & -0.02 & $<0.01$ & 0.11 \\
\hline 9 & Parts A \& B spending [per \$100] month -2 & 0.00 & $<0.01$ & 0.11 \\
\hline 10 & NAA usage gap month -4 & 0.06 & 0.01 & 0.12 \\
\hline 11 & Number of unique physicians seen month 0 & 0.01 & $<0.01$ & 0.12 \\
\hline 12 & Hospital discharge month -1 & 0.05 & 0.01 & 0.12 \\
\hline 13 & Hospital discharge month -4 & 0.04 & 0.01 & 0.12 \\
\hline 14 & Part D spending [per \$100] month 0 & -0.00 & $<0.01$ & 0.12 \\
\hline 15 & Part D spending [per \$100] month -3 & 0.00 & $<0.01$ & 0.12 \\
\hline 16 & Hypoglycemia month -1 & 0.17 & 0.03 & 0.13 \\
\hline 17 & Discontinuance of ACEIs/ARBs month -1 & 0.15 & 0.02 & 0.13 \\
\hline 18 & Parts A \& B spending [per \$100] month -3 & 0.00 & $<0.01$ & 0.13 \\
\hline 19 & LIS (1: yes, $0:$ no) & 0.01 & $<0.01$ & 0.13 \\
\hline 20 & Discontinuance of statins month -1 & 0.13 & 0.02 & 0.13 \\
\hline 21 & SNF discharge month -1 & 0.10 & 0.02 & 0.13 \\
\hline 22 & Discontinuance of ACEIs/ARBs month -2 & 0.10 & 0.02 & 0.13 \\
\hline 23 & Hypoglycemia month 0 & 0.14 & 0.03 & 0.13 \\
\hline 24 & Count of ED visits month -2 & 0.02 & $<0.01$ & 0.13 \\
\hline 25 & Eye exam month -1 & -0.01 & $<0.01$ & 0.13 \\
\hline 26 & ESRD month 0 & 0.05 & 0.01 & 0.13 \\
\hline 27 & Uncontrolled diabetes month -1 & 0.02 & $<0.01$ & 0.13 \\
\hline 28 & SNF discharge month -4 & 0.06 & 0.02 & 0.13 \\
\hline 29 & Discontinuance of statins month -2 & 0.08 & 0.02 & 0.13 \\
\hline 30 & Eye exam month 0 & -0.01 & $<0.01$ & 0.13 \\
\hline 31 & Insulin use month -3 & -0.02 & 0.01 & 0.13 \\
\hline 32 & RxHCC count & 0.00 & $<0.01$ & 0.13 \\
\hline 33 & Black race & 0.01 & $<0.01$ & 0.13 \\
\hline 34 & Other race & -0.00 & $<0.01$ & 0.13 \\
\hline 34 & Cancer month -3 & -0.02 & 0.01 & 0.13 \\
\hline 35 & Parts A \& B spending [per \$100] month -1 & 0.00 & $<0.01$ & 0.14 \\
\hline 36 & Eye exam month -2 & -0.01 & $<0.01$ & 0.14 \\
\hline 37 & SNF discharge month -3 & 0.05 & 0.02 & 0.14 \\
\hline 38 & Hypoglycemia month -2 & 0.08 & 0.03 & 0.14 \\
\hline 39 & Alc test month -2 & -0.01 & $<0.01$ & 0.14 \\
\hline 40 & Uncontrolled diabetes month -4 & -0.01 & $<0.01$ & 0.14 \\
\hline 41 & Part D spending [per \$100] month -4 & 0.00 & $<0.01$ & 0.14 \\
\hline 42 & Discontinuance of statins month -3 & 0.07 & 0.03 & 0.14 \\
\hline 43 & SNF discharge month 0 & 0.05 & 0.02 & 0.14 \\
\hline 44 & Number of NAAs filled month -4 & -0.00 & $<0.01$ & 0.14 \\
\hline 45 & Number of NAAs filled month -3 & 0.00 & $<0.01$ & 0.14 \\
\hline 46 & Long-term complications month -1 & 0.01 & 0.01 & 0.14 \\
\hline 47 & Hypoglycemia month -4 & 0.07 & 0.03 & 0.14 \\
\hline 48 & Eye exam month -3 & -0.01 & $<0.01$ & 0.14 \\
\hline 49 & Glucose test month -4 & -0.01 & 0.01 & 0.14 \\
\hline 50 & Hospice admission month 0 & 0.09 & 0.04 & 0.14 \\
\hline
\end{tabular}

Note: Variables entered sequentially based on lowest $P$ value among remaining variables until no remaining variable was statistically significant at $P<0.05$. Coefficient values shown are from the final model iteration.

$A 1 c=$ hemoglobin $A 1 c ; A C E I=$ angiotensin-converting enzyme inhibitor; $A R B=$ angiotensin receptor blockers; $E D=$ emergency department; ESRD = end-stage renal disease; LIS =low-income subsidy; NAA = noninsulin antihyperglycemic agents; SNF=skilled nursing facility. 
Obviously, this approach does not work going forward, when it is unknown if or when a person will discontinue therapy. For example, we found that discontinuing statins raised the probability of NAA discontinuance by $37 \%$ in the index month, $13 \%$ in month $-1,8 \%$ in month -2 , and $7 \%$ in month $-3 \%$. A clinician looking forward in time would thus not know whether a patient observed to discontinue statins had a $7 \%$ or a $37 \%$ chance of NAA discontinuation in the next month.

One approach to address this issue would be to establish contemporary correlates with each timed measure of statin discontinuance (and by extension other proximal predictor variables) to characterize the particular collection of factors uniquely associated with each pre-index month and then prospectively test the sensitivity and specificity of the results in predicting drug discontinuance in another dataset. Such an exercise is beyond the scope of this study but offers promise as a tool for prospectively assessing medication persistence.

A good prognostic tool will require an expanded set of predictor variables. Even though we have shown that dynamic variables are much more predictive of medication discontinuance than static factors, the fact remains that we only explained $14 \%$ of the variance. Like most adherence studies that rely on claims data, we did not directly observe important patient behaviors that could trigger drug discontinuance. There is voluminous literature on patient beliefs and behaviors that could theoretically be brought to bear on this subject with the right data. ${ }^{5-7,10,13}$ It is equally important to identify drug discontinuation initiated by prescribers, but without direct information on prescription orders (original prescriptions, refills, and stop orders), this is impossible to achieve. Eventually, when claims data are routinely combined with electronic medical records, it will become possible to examine a more expansive set of proximal reasons for drug discontinuation, including patient intolerance, side effects, and lack of drug effectiveness.

Another limitation of our approach is that we focused exclusively on long-term NAA discontinuers $(12+$ months with no observed use) with the assumption that individuals meeting this criterion had ceased taking this class of diabetes medications altogether, as opposed to those taking a drug holiday or otherwise experiencing short gaps in therapy. However, we discovered that, among study subjects whom we could track up to 24 months after discontinuation, approximately a quarter resumed NAA use (results not shown) with unobserved future resumption rates presumably higher still. This suggests that adherence studies focused on persistence with chronic medications are mostly about short and long gaps in adherence rather than outright discontinuance, even if that goes unrecognized in studies of short duration. What our research adds to this line of reasoning is that different proximal factors may be associated with short and long gaps and that what we have been able to deduce about predictors of long-term gaps may or may not hold for shorter gaps.

A final limitation is that we focused on diabetics taking a single class of medications and cannot generalize the findings to other chronic diseases or other evidence-based drugs. Filling this knowledge gap should be given high priority in future medication adherence studies.

\section{Conclusions}

In this study, a cascade of dynamic changes in drug use, disease severity, and institutional episodes have been observed preceding discontinuance with NAA therapy among Medicare Part D enrollees with diabetes between 2006 and 2008. Understanding that lack of persistence in drug use is a dynamic rather than a static phenomenon opens up new avenues for investigating and ultimately improving adherence behavior among older adults.

\section{Authors}

BRUCE C. STUART, PhD; XIAN SHEN, MS; NICOLE BRANDT, PharmD; F. ELLEN LOH, BSPharm, MBA; FRANKLIN HENDRICK, BS; and CAROLINE KIM, BS, University of Maryland School of Pharmacy, Baltimore, Maryland. CHARLENE C. QUINN, RN, PhD, FAAN, University of Maryland School of Medicine, Baltimore, Maryland, and PAMELA ROBERTO, MPP, Pharmaceutical Research and Manufacturers of America, Washington, DC, and University of Maryland School of Pharmacy, Baltimore, Maryland. XINGYUE HUANG, PhD, and SWAPNIL RAJPATHAK, MD, Merck Research Labs, Merck \& Co., Kenilworth, New Jersey.

AUTHOR CORRESPONDENCE: Xian Shen, MS, University of Maryland School of Pharmacy, 220 Arch St., 12th Fl., Baltimore, MD 21201. Tel.: 410.706.1418; Fax: 410.706.5394;

E-mail: xshen@umaryland.edu.

\section{DISCLOSURES}

This study was funded by Merck \& Co. Huang and Raipathak are employees of Merck \& Co. Brandt reports consultancy and speaker fees from Catapult, Omnicare, RAND, HRSA, CMS, and AGS Beers Criteria. Loh is currently employed at Touro College of Pharmacy. All other authors have no relevant potential conflicts of interest to disclose.

Study concept and design were primarily contributed by Stuart, Quinn, and Brandt, along with Shen, Roberto, Hendrick, Huang, and Rajpathak. Shen, Loh, Hendrick, and Kim collected the data, and data interpretation was performed primarily by Stuart, Shen, and Roberto, assisted by Quinn, Brandt, Hendrick, Huang, and Rajpathak. The manuscript was written primarily by Stuart, with assistance from the other authors, and revised by Huang, Rajpathak, and Stuart, with assistance from the other authors. 


\section{REFERENCES}

1. Selvin E, Marinopoulos S, Berkenblit G, et al. Meta-analysis: glycosylated hemoglobin and cardiovascular disease in diabetes mellitus. Ann Intern Med. 2004;141(6):421-31.

2. Brown AF, Mangione CM, Saliba D, et al. California Healthcare Foundation/American Geriatrics Society Panel on Improving Care for Elders with Diabetes: guidelines for improving the care of the older person with diabetes mellitus. J Am Geri Soc. 2003;51(5 Suppl Guidelines):S265-80.

3. Vanelli M, Pedan A, Liu N, Hoar J, Messier D, Kiarsis K. The role of patient inexperience in medication discontinuation: a retrospective analysis of medication nonpersistence in seven chronic illnesses. Clin Ther. 2009;31(11):2628-52.

4. Zhang Q, Zhao C, Davies MJ, Radican L, Seck T. Compliance and persistence with concomitant statin and oral antihyperglycemic therapy. Am J Manag Care. 2011;17(11):746-52.

5. DiMatteo MR. Variations in patients' adherence to medical recommendations: a quantitative review of 50 years of research. Med Care. 2004:42(3):200-09.

6. Cramer JA, Benedict A, Muszbec N, Keskinaslan A, Khan ZM. The significance of compliance and persistence in the treatment of diabetes, hypertension, and dyslipidemia: a review. Int J Clin Pract. 2008;62(1):76-87.

7. Gellad WF, Grenard J, McGlynn EA. A review of barriers to medication adherence: a framework for driving policy options. Santa Monica, CA: RAND Corporation; 2009. Available at: http://www.rand.org/pubs/technical_reports/TR765.html. Accessed July 27, 2016.

8. Rathmann W, Kostev K, Gruenberger JB, Dworak M, Bader G, Giani G. Treatment persistence, hypoglycaemia and clinical outcomes in type 2 diabetes patients with dipeptidl peptidase- 4 inhibitors and sulphonylureas: a primary database analysis. Diab Obes Metab. 2013;15(1):55-61.

9. Bron M, Marynchenko M, Yang H, Yu AP, Wu EQ. Hypoglycemia, treatment discontinuation, and costs with type 2 diabetes mellitus on oral antidiabetic drugs. Postgrad Med. 2012;124(1):2525-64.

10. Seabury SE, Gupta CN, Philipson, TJ, Henkhaus LE; PhRMA Medication Adherence Advisory Council. Understanding and overcoming barriers to medication adherence: a review of research priorities. J Manag Care Spec Pharm. 2014;20(8):775-83. Available at: http://www.jmcp.org/doi/10.18553/ jmcp.2014.20.8.775.
11. Yeaw J, Benner JS, Walt JG, Sian S, Smith DB. Comparing adherence and persistence across 6 chronic medication classes. J Manag Care Pharm. 2009;15(9):728-40. Available at: http://www.jmcp.org/doi/10.18553/ jmcp.2009.15.9.728.

12. Hincapie AL, Taylor AM, Boesen KP, Warholak T. Understanding reasons for nonadherence to medications in a Medicare Part D beneficiary sample. J Manag Care Spec Pharm. 2015;21(5):391-99. Available at: http:// www.jmcp.org/doi/10.18553/jmcp.2015.21.5.391.

13. Touchette DR, Shapiro NL. Medication compliance, adherence, and persistence: current status of behavioral and educational interventions to improve outcomes. J Manag Care Pharm. 2008;14(6 Suppl S-d):S2-S10. Available at: http://www.amcp.org/data/jmcp/Aug08\%20Suppl\%20D_S2-S10.pdf.

14. Jonikas MA, Mandl KD. Surveillance of medication use: early identification of poor adherence. J Am Med Inform Assoc. 2012;19(4):649-54.

15. Chang A, Liberman JN, Coulen C, Berger JE, Brennan TA. Value-based insurance design and antidiabetic medication adherence. Am J Man Care. 2010;2(1):39-44. Available at: http://www.ajpb.com/journals/ajpb/2010/ vol2_nol/value-based-insurance-design-and-antidiabetic-medication-adherence. Accessed July 27, 2016

16. Ramachandran A, Winter M, Mann DM. Association of visit-to-visit variability of hemoglobin Alc and medication adherence. J Manag Care Spec Pharm. 2015;21(3):229-37. Available at: http://www.jmcp.org/doi/10.18553/ jmcp.2015.21.3.229.

17. Iyengar RN, Balagere DS, Henderson RR, LeFrancois AL, Rabbitt RM, Frazee SG. Association between dispensing channel and medication adherence among Medicare beneficiaries taking medications to treat diabetes, high blood pressure, and high blood cholesterol. J Manag Care Spec Pharm. 2014;20(8):851-61. Available at: http://www.jmcp.org/doi/10.18553/ jmcp.2014.20.8.851.

18. Sacks NC, Burgess JF, Cabral HJ, McDonnell ME, Pizer SD. The effects of cost sharing on adherence to medications prescribed for concurrent use: do definitions matter? J Manag Care Spec Pharm. 2015;21(8):678-87. Available at: http://www.jmcp.org/doi/10.18553/jmcp.2015.21.8.678.

19. Medicare Payment Advisory Commission. Report to the Congress: Medicare payment policy. March 2011. Available at: http://www.medpac.gov/ documents/reports/marl1_entirereport.pdf?sfvrsn=0. Accessed July 27, 2016.

20. Wooldridge JM. Introductory Econometrics: A Modern Approach. 5th ed. Mason, $\mathrm{OH}$ : Thomson; 2013. 


\section{APPENDIX A Logistic Regression Results Predicting NAA Discontinuation with Static Variables Only}

Static Variable

\begin{tabular}{|r|r} 
Odds Ratio \\
\hline
\end{tabular}

Age (per additional year)

Male (ref = female)

1.0036

1.0512

$95 \%$ CI

Race (ref=white)

\begin{tabular}{|c|c|c|}
\hline Black & 1.3413 & $(1.2511-1.4379)$ \\
\hline Other race & 0.9955 & $(0.9121-1.0866)$ \\
\hline Old age as original reason for entitlement (ref=SSDI or ESRD) & 0.8358 & $(0.777-0.8992)$ \\
\hline LIS (ref=no LIS) & 1.1370 & $(1.0148-1.274)$ \\
\hline Dual eligible (ref=not dual eligible) & 0.9896 & $(0.8876-1.1032)$ \\
\hline RxHCC (per additional RxHCC) & 1.0995 & $(1.0914-1.1076)$ \\
\hline C-statistic & & 0.6003 \\
\hline
\end{tabular}

CI=confidence interval; ESRD = end-stage renal disease; LIS = low-income subsidy; NAA = noninsulin antihyperglycemic agents; ref = reference; RxHCC = Prescription Drug Hierarchical Condition Category; SSDI = Social Security Disability Insurance. 


\section{APPENDIX B Stepwise Logistic Regression Results Predicting NAA Discontinuation as a} Function of Static and Dynamic Variables

\begin{tabular}{|c|c|c|c|}
\hline Step & Variable & Odds Ratio & $95 \%$ CI \\
\hline 1 & Insulin use in month 0 & 2.9870 & $(2.807-3.177)$ \\
\hline 2 & Discontinuance of statins in month 0 & 9.7680 & $(7.971-11.97)$ \\
\hline 3 & Number of NAAs filled in month -2 & 0.6640 & $(0.633-0.696)$ \\
\hline 4 & Number of unique physicians seen in month -1 & 1.0300 & $(1.017-1.044)$ \\
\hline 5 & Discontinuance of ACEIs/ARBs in month 0 & 8.7520 & $(7.242-10.576)$ \\
\hline 6 & Parts A \& B spending [per \$100] in month -2 & 1.0010 & $(1-1.002)$ \\
\hline 7 & NAA usage gap in month -4 & 1.5960 & $(1.45-1.757)$ \\
\hline 8 & NAA usage gap in month -1 & 0.2310 & $(0.197-0.272)$ \\
\hline 9 & Number of NAAs filled in month -1 & 0.7040 & $(0.671-0.738)$ \\
\hline 10 & NAA usage gap in month -2 & 2.1510 & $(1.944-2.381)$ \\
\hline 11 & Number of unique physicians seen in month 0 & 1.0500 & $(1.04-1.061)$ \\
\hline 12 & Hospital discharge in month -1 & 1.4980 & $(1.294-1.733)$ \\
\hline 13 & Discontinuance of ACEIs/ARBs in month -1 & 3.1740 & $(2.399-4.2)$ \\
\hline 14 & Hospital discharge in month -4 & 1.3610 & $(1.211-1.531)$ \\
\hline 15 & Hypoglycemia in month -1 & 2.8450 & $(2.051-3.947)$ \\
\hline 16 & Discontinuance of statins in month -1 & 2.9940 & $(2.187-4.098)$ \\
\hline 17 & Part D spending [per \$100] in month 0 & 0.9450 & $(0.937-0.953)$ \\
\hline 18 & Part D spending [per \$100] in month -3 & 1.0230 & $(1.015-1.031)$ \\
\hline 19 & RxHCC count & 1.0260 & $(1.017-1.036)$ \\
\hline 20 & LIS (1: yes, $0:$ no) & 1.1680 & $(1.087-1.255)$ \\
\hline 21 & Discontinuance of ACEIs/ARBs in month -2 & 2.4040 & $(1.763-3.278)$ \\
\hline 22 & Hypoglycemia in month 0 & 2.4600 & $(1.732-3.493)$ \\
\hline 23 & Parts A \& B spending [per \$100] in month -3 & 1.0010 & $(1-1.001)$ \\
\hline 24 & Count of ED visits in month -2 & 1.1220 & $(1.044-1.207)$ \\
\hline 25 & Discontinuance of statins in month -2 & 2.1470 & $(1.502-3.069)$ \\
\hline 26 & Number of NAAs filled in month -4 & 0.8910 & $(0.849-0.935)$ \\
\hline 27 & Part D spending [per \$100] in month -4 & 1.0130 & $(1.005-1.021)$ \\
\hline 28 & SNF discharge in month 0 & 1.5280 & $(1.199-1.948)$ \\
\hline 29 & SNF discharge in month -1 & 1.3940 & $(1.121-1.734)$ \\
\hline 30 & ESRD in month 0 & 1.3810 & $(1.154-1.652)$ \\
\hline 31 & Uncontrolled diabetes in month -1 & 1.2160 & $(1.11-1.331)$ \\
\hline 32 & Eye exam in month -1 & 0.8350 & $(0.743-0.94)$ \\
\hline 33 & Discontinuance of statins in month -3 & 1.8750 & $(1.278-2.752)$ \\
\hline 34 & Hypoglycemia in month -4 & 1.7230 & $(1.146-2.589)$ \\
\hline \multirow{2}{*}{35} & Black race & 1.1210 & $(1.038-1.211)$ \\
\hline & Other race & 0.9470 & $(0.861-1.041)$ \\
\hline 36 & SNF discharge in month -4 & 1.4000 & $(1.084-1.809)$ \\
\hline 37 & Eye exam in month -2 & 0.8560 & $(0.762-0.962)$ \\
\hline 38 & Hospital discharge in month -2 & 1.2090 & (1.038-1.408) \\
\hline 39 & Eye exam in month 0 & 0.8570 & $(0.765-0.962)$ \\
\hline 40 & Uncontrolled diabetes in month -4 & 0.8770 & $(0.793-0.971)$ \\
\hline 41 & Alc test in month -2 & 0.9040 & $(0.84-0.974)$ \\
\hline 42 & Hypoglycemia in month -2 & 1.6290 & $(1.12-2.37)$ \\
\hline 43 & SNF discharge in month -3 & 1.3050 & $(1.02-1.669)$ \\
\hline 44 & Cancer in month -3 & 0.7680 & $(0.622-0.947)$ \\
\hline 45 & Hospice admission in month 0 & 1.7790 & $(1.066-2.971)$ \\
\hline 46 & Parts A \& B spending [per \$100] in month -1 & 1.0010 & $(1-1.002)$ \\
\hline 47 & Eye exam in month -3 & 0.8800 & $(0.784-0.988)$ \\
\hline 48 & Age & 1.0050 & $(1.002-1.008)$ \\
\hline 49 & Old age as original reason for Medicare entitlement & 0.9190 & $(0.848-0.995)$ \\
\hline 50 & Part D spending [per \$100] in month -1 & 1.0080 & $(1-1.016)$ \\
\hline
\end{tabular}

$A 1 c=$ hemoglobin $A 1 c ; A C E I=$ angiotensin-converting enzyme inhibitor; $A R B=$ angiotensin receptor blockers; $C I=$ confidence interval; $E D=$ emergency department; $E S R D=$ end-stage renal disease; LIS = low-income subsidy; NAA = noninsulin antihyperglycemic agents; RxHCC = Prescription Drug Hierarchical Condition Category; SNF = skilled nursing facility. 